\title{
INITIAL-BOUNDARY VALUE PROBLEMS FOR HYPERBOLIC SYSTEMS IN REGIONS WITH CORNERS. I(1)
}

BY

\author{
STANLEY OSHER
}

\begin{abstract}
In recent papers Kreiss and others have shown that initialboundary value problems for strictly hyperbolic systems in regions with smooth boundaries are well-posed under uniform Lopatinskiil conditions. In the present paper the author obtains new conditions which are necessary for existence and sufficient for uniqueness and for certain energy estimates to be valid for such equations in regions with corners. The key tool is the construction of a symmetrizer which satisfies an operator valued differential equation.
\end{abstract}

I. Introduction. The aim of this series of papers will be to obtain energy estimates for mixed initial-boundary value problems for certain hyperbolic partial differential equations in regions with corners. The work will revolve around the introduction of a new symmetrizer for general initial-boundary value problems. This symmetrizer seems to have a significance of its own.

Kreiss [6] has recently shown that a general type of mixed initial-boundary value problem in regions with a smooth boundary for a strictly hyperbolic operator with variable real coefficients is well-posed in the $L_{2}$ norm. His result was improved by Ralston [10], to permit complex valued coefficients, and by Rauch [11], to obtain a semigroup estimate. Sakomoto [12] independently obtained similar results for a single higher order equation. Earlier work was done by Hersh [3], who obtained classical existence and uniqueness results for equations with constant coefficients without obtaining energy estimates. An important new idea in Kreiss' work came in the nature of the symmetrizer he used in order to obtain his estimate.

The symmetrizer we shall introduce below involves certain solutions to a homogeneous differential equation. These are the same exponential solutions that appear in the Kreiss condition for well-posedness, and indeed which appear in the analogous Lopatinskii condition for elliptic systems [8]. We shall, in a later paper, use the symmetrizer introduced here to obtain special cases of Kreiss' result in a simpler fashion.

Received by the editors December 27, 1971.

AMS (MOS) subject classifications (1970). Primary 35L50, 35L30; Secondary 78A45.

Key words and phrases. Hyperbolic equations, initial boundary conditions, symmetrizer, energy estimate, well-posedness.

(1) Research partially supported under N.S.F. Grant GP29-273. 
In the regions with corners discussed below, Kreiss condition for each half space problem need not imply well-posedness of the corner problem. We shall give a simple counterexample below. We introduce a new condition which is both natural and necessary in the sense that if a solution for constant coefficients does exist, then we are led directly to the condition in a natural way, and we can write down the answer in this case. We then construct a new norm, with which an a priori estimate is obtained, at least for constant coefficients. In order to do problems with variable coefficients, we shall show in a future paper that this symmetrizer has certain smoothness properties, and hence the functional calculus for pseudo-differential operators applies. It should be noted that in the elliptic case, modulo a weakening of the norm at the corner, Kondrat' ev [4] has shown that an elliptic boundary value problem remains such near a corner, in distinction to what happens here. We discuss this in the last section.

The situation encountered here appears to be analogous to that found in the study of Toeplitz operators in two dimensions, e.g. Douglas and Howe [2], where invertibility of the two half space problems is necessary, but not sufficient, for invertibility of the corner problem.

Special hyperbolic differential equations in such regions have been studied in connection with water waves over sloping beaches, e.g. Peters [9], and in wedge problems in optics, e.g. Kraus and Levine [5]. Recently, Kupka and the author [7], used a new Weiner-Hopf technique to obtain a closed form solution to such a problem.

II. Statement of the problem; results. We begin by considering the hyperbolic system with constant coefficients,

$$
A u_{x}+B u_{y}+\sum_{j=3}^{n} C_{j} u_{z}+D u-u_{t}=F(x, y, z, t),
$$

where $z=\left(z_{3}, z_{4}, \cdots, z_{n}\right), u$ and $F$ are complex valued $m$ vectors and the system is hyperbolic in the sense that for the $m \times m$ matrices $A, B, C_{j}$, the matrix

$$
\operatorname{det}[A i \xi+B i \eta+C i \omega-\lambda]=0
$$

has only purely imaginary roots.

Here $C i \omega=\sum_{j=3}^{n} C_{j} i \omega_{j}, \omega=\left(\omega_{3}, \cdots, \omega_{n}\right)$, and $\xi, \eta, \omega$ are real, with $|\xi|^{2}+$ $|\eta|^{2}+|\omega|^{2}>0$. (We shall always use the convention that || of a vector is the square root of the sum of the squares of its components.)

We $w$ ish to solve this problem in the region:

$$
x, y, t \geq 0 ; \quad-\infty<z_{j}<\infty, \quad j=3,4, \cdots, n,
$$

with initial conditions

$$
u(x, y, z, 0)=0
$$


We further assume that

$$
A=\left(\begin{array}{ccccc}
a_{1} & & 0 & \cdots & 0 \\
\cdot & \ddots & & & \cdot \\
\cdot & & a_{l} & & \cdot \\
\cdot & & & a_{l+1} & \cdot \\
\cdot & & & & \cdot \\
0 & \ldots & 0 & & a_{m}
\end{array}\right) \text {, where } \begin{gathered}
a_{j}<0, j=1,2, \ldots, l, \\
a_{k}>0, k=l+1, \cdots, m,
\end{gathered}
$$

and there exists a nonsingular matrix $T$ such that

$$
T B T^{-1}=\left(\begin{array}{ccccc}
b_{1} & & 0 & \cdots & 0 \\
\cdot & \ddots & & & \cdot \\
\cdot & & b_{p} & & \cdot \\
\cdot & & & b_{p+1} & \cdot \\
\cdot & & & \ddots & \cdot \\
n & \ldots & 0 & & b_{m}
\end{array}\right) \text {, where } \begin{aligned}
& b_{j}<0, j=1,2, \cdots, p, \\
& b_{k}>0, k=p+1, \cdots, m .
\end{aligned}
$$

We now impose boundary conditions of the form

$$
u^{\mathrm{I}}(0, y, z, t)-S u^{\mathrm{II}}(0, y, z, t)=g(y, z, t),
$$

(b) $\quad(T u)^{\mathrm{III}}(x, n, z, t)-R(T u)^{\mathrm{IV}}(x, 0, z, t)=b(x, z, t)$,

where

$$
\begin{aligned}
u^{\mathrm{I}} & =\left(u_{1}, \cdots, u_{l}\right)^{T}, \quad u^{\mathrm{II}}=\left(u_{l+1}, \cdots, u_{m}\right)^{T}, \\
u^{\mathrm{III}} & =\left(u_{1}, \cdots, u_{p}\right)^{T}, \quad u^{\mathrm{IV}}=\left(u_{p+1}, \cdots, u_{m}\right)^{T} .
\end{aligned}
$$

$S$ and $R$ are constant matrices having $l$ rows and $(m-l)$ columns, and $p$ rows, $(m-p)$ columns respectively.

It is clear from finite speed of propagation considerations, that in order for the corner problem to be well-posed in any reasonable sense, it is necessary that the two half space problems:

(a)

Equation (2.1) in region $0 \leq x, t<\infty,-\infty<y, z_{j}<\infty$, and

$$
\text { Equation (2.1) in region } 0 \leq y, t<\infty,-\infty<x, z_{j}<\infty \text {, }
$$
with boundary condition (2.6(b))

be well-posed.

We first Laplace transform (2.1) in time and use (2.3). In problem (a), Fourier transform in $y$ and all the $z_{j}$ variables. We have, if $F \equiv 0$,

$$
u_{x}+A^{-1}\left(B i \omega_{2}+i C(\omega)-s\right) u=0, \quad x \geq 0 .
$$

[It is easily shown that the matrix $D$ plays only a trivial role in this problem and may be set equal to zero throughout.] 
It is easily seen that Kreiss' condition is valid for both half spaces for all $\omega_{2}, \omega$ are real, $s=\eta+i \xi \quad$ with $\eta>0, \xi$ real.

We can show (e.g. [3]), that this ordinary differential equation has exactly $l$ linearly independent solutions which decay exponentially as $x \rightarrow+\infty$. Normalize them at $x=0$, and obtain $l$ functions

$$
\Phi_{j}\left(x, \omega_{2}, \omega, s\right), \quad j=1,2, \cdots, l .
$$

Kreiss' condition is, at $x=0$,

$$
\text { determinant }[I,-S]\left[\Phi_{1}, \cdots, \Phi_{l}\right] \neq 0
$$

for all $\omega_{2}, \omega, s$, with Real $s \geq 0$.

This condition is natural in that if one wishes to solve (2.7) with the transformed boundary condition of $(2.6(a))$, the inverse of this matrix must appear in the solution.

Kreiss' condition for problem (b) is completely analogous.

We make an assumption.

(2.1) Assumption. Kreiss' condition is valid for problems (a) and (b).

We now proceed by first assuming that a solution exists to (2.1), (2.6) with $F=0, b=0$, and then seeing what this assumption leads to. We have

$$
A u_{x}+B u_{y}+(C i \omega-s) u=0, \quad x, y \geq 0 .
$$

$$
u^{\mathrm{I}}(0, y, \omega, s)-S u^{\mathrm{II}}(\cap, y, \omega, s)=g(y, \omega, s),
$$

(b) $\quad(T u)^{\mathrm{III}}(x, 0, \omega, s)-R(T u)^{\mathrm{IV}}(x, \cap, \omega, s)=b(x, \omega, s)$.

Let $v$ be the function defined for $y \geq 0,-\infty<x<\infty$ as

$$
\begin{gathered}
v(x, y, \omega, s)=u(x, y, \omega, s) \quad \text { if } \cap \leq x<\infty, \\
v(x, y, \omega, s)=\cap \quad \text { if }-\infty<x<c .
\end{gathered}
$$

Then $v$ satisfies

$$
A v_{x}+B v_{y}+(C i \omega-s) v=A \delta(x-0) u_{+}(0, y, \omega, s), \quad y \geq 0,
$$

with boundary conditions

$$
(T v)^{\mathrm{III}}(x, 0, \omega, s)-R(T v)^{\mathrm{IV}}(x, 0, \omega, s)=0, \quad-\infty<x<\infty .
$$

We know that

$$
u_{+}(0, y, \omega, s)=\left(\begin{array}{c}
s \Phi(y, \omega, s) \\
\Phi(y, \omega, s)
\end{array}\right)+\left(\begin{array}{c}
g(y, \omega, s) \\
0
\end{array}\right) .
$$

If we Fourier transform (2.11) in $x$, the resulting equation

$$
v_{y}+B^{-1}\left(A i \omega_{1}+C i \omega-s\right) v=u_{+}(0, y, \omega, s),
$$

with boundary conditions 


$$
(T v)^{\mathrm{III}}\left(\omega_{1}, 0, \omega, s\right)-R(T \nu)^{\mathrm{IV}}\left(\omega_{1}, 0, \omega, s\right)=0,
$$

can be solved uniquely, using Kreiss' condition, if we require that $u_{+}(0, y, \omega, s)$ be smooth with compact support and the solution not increase exponentially as $y \rightarrow+\infty$. We thus have $v(x, y, \omega, s)$, and hence $v(x, 0, \omega, s)$ for $x \geq 0$, which must equal $u(x, 0, \omega, s)$ for $x \geq 0$.

We next let $w$ be the function defined for $x \geq 0,-\infty<y<\infty$ with

Then $w$ obeys

$$
\begin{gathered}
w(x, y, \omega, s)=u(x, y, \omega, s) \quad \text { if } 0 \leq y<\infty, \\
w(x, y, \omega, s)=0 \text { if }-\infty<y<0 .
\end{gathered}
$$

$$
\begin{array}{ll}
A w_{x}+B w_{y}+(C i \omega-s) w=B \delta(y-0) v(x, 0, \omega, s), \\
x \geq 0, \quad \text { with boundary conditions } \\
w^{\mathrm{I}}(0, y, \omega, s)-S w^{\mathrm{II}}(0, y, \omega, s)=g(y, \omega, s) & \text { if } y \geq 0, \\
w^{\mathrm{I}}(0, y, \omega, s)-S w^{\mathrm{II}}(0, y, \omega, s)=0 & \text { if } y<0 .
\end{array}
$$

We can Fourier transform in $y$ and proceed to solve this in the right half plane in the analogous fashion as above, finally obtaining $w(0, y, \omega, s)$. If a solution does indeed exist to this problem, then it must be true that

$$
w(0, y, \omega, s)=\left(\begin{array}{c}
S \Phi(y, \omega, s) \\
\Phi(y, \omega, s)
\end{array}\right)+\left(\begin{array}{c}
g(y, \omega, s) \\
0
\end{array}\right)
$$

however, we have constructed this function such that

$$
u(0, y, \omega, s)=\left(\begin{array}{c}
S\left(T_{\omega, s} \Phi+P_{\omega, s} g\right) \\
T_{\omega, s} \Phi+P_{\omega, s} g
\end{array}\right)+\left(\begin{array}{c}
g(y, \omega, s) \\
0
\end{array}\right)
$$

where $T_{\omega, s}$ and $P_{\omega_{s} s}$ are linear operators which will be discussed in more detail in succeeding sections. Thus, if a solution to the corner problem exists, it is necessary that the equation

$$
\left[T_{\omega, s}-I\right] \Phi=-P_{\omega, s} g
$$

has a solution for all $\omega, s$. We introduce the convention here that all $\omega_{j}$, $s$ mean all real $\omega_{j}$ and all $s$ with real part positive. Moreover, if we expect to bound the solution on the boundary in terms of some norm of $g$, we wish the left inverse to be bounded in some sense. Finally, if this is all valid, then we need only use the value of $\Phi$ here obtained in (2.13), then solve $\left(2.11^{\prime}\right),\left(2.12^{\prime}\right)$, to obtain the correct answer.

We pause here for an example of this condition. Consider the equation

$$
\left[\begin{array}{cc}
-1 & 0 \\
0 & 1
\end{array}\right] u_{x}+\left[\begin{array}{cc}
1 & 0 \\
0 & -1
\end{array}\right] u_{y}-s u=0
$$

in $x, y \geq 0$, with boundary conditions 


$$
u_{1}(0, y, s)-a u_{2}(0, y, s)=f(y, s), \quad u_{2}(x, 0, s)-b u_{1}(x, 0, s)=0 .
$$

It is easily seen that Kreiss' condition is valid for both half spaces for all complex $a$ and $b$. A simple calculation shows that

$$
T_{s} \Phi=e^{-2 s y} a b \Phi
$$

and in fact that if a solution exists, it must be

$$
\begin{aligned}
& u_{1}(x, y, s)=\frac{e^{-s x} f(x+y, s)}{1-a b e^{-2 s(x+y)}} \\
& u_{2}(x, y, s)=\frac{b e^{s y} e^{-s(x+y)} f(x+y, s)}{1-a b e^{-2 s(x+y)}}
\end{aligned}
$$

and we see that an a priori estimate of the type sought below is possible if and only if $|a b|<1$, which is exactly the condition that $I-T_{s}$ have a uniformly bounded left inverse.

We now state our remaining definitions, assumptions, and results.

(2.2) Assumption. The matrices $A, B, C_{j}$ are symmetric, constant, and complex valued. Thus the matrix $T$ in (2.5) can be taken to be unitary.

(2.3) Assumption. Either the operator is strictly hyperbolic in the sense that the eigenvalues in (2.2) are distinct, or the following conditions are true:

(a) There exist uniformly bounded and invertible matrix functions $U\left(\omega_{2}, s, \omega\right)$, $V\left(\omega_{1}, s, \omega\right)$ both of which are $C^{\infty}$ with $C^{\infty}$ uniformly bounded inverse for $\operatorname{Re} s \geq$ $K>0$, all $\omega_{j}, \omega$, for any $K>0$. Moreover, $\exists$ a constant $\delta_{1}>0$ with

$$
U^{-1} A^{-1}\left(B i \omega_{2}+\bar{s}+C i \omega\right) U=\left(\begin{array}{cc}
L_{11} & L_{12} \\
0 & L_{22}
\end{array}\right),
$$

with $\operatorname{Re} L_{11} \geq \delta_{1} \eta, \operatorname{Re} L_{22} \leq-\delta_{1} \eta$

$$
V^{-1} B^{-1}\left(A i \omega_{1}+\bar{s}+C i \omega\right) V=\left(\begin{array}{cc}
K_{11} & K_{12} \\
0 & K_{22}
\end{array}\right) \text {, }
$$

$\operatorname{Re} K_{11} \geq \delta_{1} \eta, \operatorname{Re} K_{22} \leq-\delta_{2} \eta$.

(2.4) Assumption. Any real eigenvalue of $A^{-1} B$ is negative.

Notice this implies that $l+p=m$.

Le mma (4.1) below will show that strict hyperbolicity is sufficient for (2.24) and $(2.25)$ to be valid. Of course, it is not necessary, e.g. the operator defined in (2.19).

The next assumption is the critical one.

(2.5) Assumption. The equation

$$
\left(I-T_{\omega, s}\right) \Phi=g
$$

posesses a solution $\Phi$ for any $g(y)$ which is smooth with bounded support, having 
the property

(2.27)(a) $\int_{0}^{\infty}|\phi(y, \omega, s)|^{2} d y \leq K_{1} \int_{0}^{\infty} \sum_{p=0}^{K_{2}}\left|\left(y \frac{\partial}{\partial y}\right)^{p} g(y)\right|^{2} d y\left(1+|\omega|^{2}+|s|^{2}\right)^{K_{3}}$,

for all real $\omega, s=\eta+i \xi$, with $\eta>0$ where the estimate is true, independent of $\omega, s$ and $g$ with $K_{1}>0, K_{2} \geq 0, K_{3}$ real, all universal constants.

We shall call this left inverse $\left(I-T_{\omega, s}\right)^{-1}$ and for technical reasons we ass ume

$$
\int_{0}^{\infty}\left|\left(y \frac{\partial}{\partial y}\right)^{j} \phi(y, \omega, s)\right|^{2} d y
$$

$$
\leq K_{1}(j) \int_{0}^{\infty} \sum_{p=0}^{K_{2}(j)}\left|\left(y \frac{\partial}{\partial y}\right)^{p} g(y)\right|^{2} d y\left(1+|\omega|^{2}+|s|^{2}\right)^{K_{3}(j)} d y
$$

for all $j_{1}=0,1,2, \cdots, \eta \geq \eta_{j}>0$, and all $\omega, \xi$. Moreover, we assume similar estimates are valid for the adjoint operator $\left(\left(I-T_{\omega, s}\right)^{-1}\right)^{*}$.

Under all these assumptions, it is not too hard to obta in our first main the orem merely by solving the equation in the manner discussed above in (2.10) to (2.18) and using some of the estimates we shall derive below.

However, this method will not be applicable in the case of variable coefficients. Moreover, the existence of a symmetrizer has an independent abstract interest.

The operators $(y(\partial / \partial y))^{p}$ appear in a somewhat disguised fashion in Kondrat'ev's paper [4] on elliptic equations in conical regions. We introduced them here for technical reasons which concern certain multiplicities of eigenvalues of the matrices in (2.24) and (2.25). We are not sure if there exist cases in which $K_{2}=0$ does not suffice, but $K_{2}>0$ does. $\left(K_{2}\right.$ is the constant appearing on the right-hand side of (2.27)(a).)

In order to check the solvability of (2.26), with estimate (2.27), we must analyze certain singular integral equations which, in general, are rather difficult to handle. However, sometimes exact solutions of these equations can be obtained, e.g. Kupka and Osher [7]. The algebra which must be checked in the Kreiss condition is often itse lf quite nontrivial. Thus, it is not surprising that the singular integral equations arising here often require special analysis. We expect to discuss this matter in a forthcoming paper.

(2.6) Definition. Let $\hat{u}(x, y, \omega, s)$ be the Fourier-Laplace transform of any vector function $u(x, y, x, t)$. We shall obtain estimates on $\int_{x, y \geq 0}|\hat{u}(x, y, \omega, s)|^{2} d x d y$ which are true for each $\omega, s$.

Define for fixed $\omega, s$

$$
\|\hat{u}\|_{P, Q}^{2}-\int_{0}^{\pi / 2} d \theta \int_{0}^{\infty}\left(1+|\omega|^{2}+|s|^{2}\right)^{Q} r d r \sum_{j=0}^{P}\left|\left(r \frac{\partial}{\partial r}\right)^{j} \hat{u}(r, \theta, \omega, s)\right|^{2},
$$


$Q$ is some real number, $P$ is a nonnegative integer, and let $((\hat{u}, \hat{v}))_{P, Q}$ be the analogous inner product.

For functions defined on the boundary, e.g. $\hat{g}(y, \omega, s)$, define

$$
|\hat{g}|_{P, Q}^{2}=\int_{0}^{\infty} \sum_{j=0}^{P}\left|\left(x \frac{\partial}{\partial x}\right)^{j} \hat{g}(x, \omega, s)\right|^{2} d x\left(1+|\omega|^{2}+|s|^{2}\right)^{Q}
$$

and $(\hat{g}, \hat{f})_{P, Q}$ is the ana logous inner product.

We also let $\hat{u}(0, y, \omega, s)=\hat{u}_{B_{1}}(y, \omega, s), \hat{u}(x, 0, \omega, s)=\hat{u}_{B_{2}}(x, \omega, s)$, and $(\hat{u}, \hat{v})$ be the inner product on $L^{2}(-\infty, \infty),((\hat{u}, \hat{v}))$ on $L_{2}[(-\infty, \infty) \times(0, \pi / 2)]$.

Main Theorem I. Under all the assumptions so far stated, there exists an a priori inequality for smooth solutions to (2.1), (2.3), (2.6) of the type (2.30) $\left(\eta-K_{3}\right)\|\hat{u}\|_{0,0}^{2}+\left|\hat{u}_{B_{1}}\right|_{0,0}^{2}+\left|\hat{u}_{B_{2}}\right|_{0,0}^{2} \leq K_{4}\|\hat{F}\|_{P, Q}^{2}+K_{5}|\hat{g}|_{P_{1}, Q_{1}}^{2}+K_{6}|\hat{b}|_{P_{2}, Q_{2}}^{2}$, for all $\eta \geq K_{3}>0$, where the $K_{j}, P, Q$, and $P_{i}, Q_{i}$ depend only on the coefficients of the equation and the boundary conditions.

In order to prove our next Main Theorem which involves the symmetrizer, we must introduce polar coordinates in the $x, y$ dependence. The transformed equation becomes, for $F=0$ :

$$
(B \cos \theta-A \sin \theta) \hat{u}_{\theta}+\left[(B \sin \theta+A \cos \theta) r \hat{u}_{r}+(C i \omega-s) r \hat{u}\right]=0 .
$$

Next, make the change of variables $r=e^{-\lambda},-\infty<\lambda<\infty$, and let $e^{-\lambda} \hat{u}=\hat{v}$. We thus have:

$$
(A \sin \theta-B \cos \theta) \hat{v}_{\theta}+(A \cos \theta+B \sin \theta) \hat{v}_{\lambda}
$$

$$
+\left[A \cos \theta+B \sin \theta+[s-C i \omega] e^{-\lambda}\right] \hat{v}=0 .
$$

Let $M(\theta, \omega, s, \cdot)$ be the differential operator defined on $L_{2}(-\infty, \infty)$ by

$$
-M(\theta, \omega, s, \cdot) \hat{v}=\left[(A \cos \theta+B \sin \theta)\left(\frac{\partial}{\partial \lambda}+1\right)+[s-C i \omega] e^{-\lambda}\right] \hat{v} \text {. }
$$

We may now state our next Main Theorem.

Main Theorem II. Under all the assumptions, there exists $\mathcal{H}$ such that $\mathcal{H}(\theta, \omega, s,).[A \sin \theta-B \cos \theta]$ is a symmetric operator valued function of $\theta, \omega, s$ on $L_{2}(-\infty, \infty)$ having the following properties:

(1) For any fixed pair of functions $\hat{u}, \hat{v}$ in $C^{\infty}(-\infty, \infty) \cap L_{2}(-\infty, \infty)$, $(\hat{v}, \mathcal{H} \hat{u})$ is a $C^{\infty}$ function of $\theta$ for fixed $\omega, s$, and each derivative is a continuous function of $\omega$ and $s$, bounded by a constant times $\left(1+|\omega|^{2}+|s|^{2}\right)$ to some power.

(2) $\mathcal{H}$ obeys the operator equation on $C^{\infty}(-\infty, \infty) \cap L_{2}(-\infty, \infty)$,

$$
\frac{1}{2} \frac{\partial}{\partial \theta}(\mathcal{H}[A \sin \theta-B \cos \theta])+\operatorname{Re} \mathcal{H}_{M}=0
$$

in fact 


$$
\mathcal{H}=(A \sin \theta-B \cos \theta) J N J^{*},
$$

with $N$ independent of $\theta$, and

$$
\frac{\partial}{\partial \theta}\left(J^{*}[A \sin \theta-B \cos \theta]\right)+J^{*} M=0 .
$$

Hence

$$
\frac{\partial}{\partial \theta}([A \sin \theta-B \cos \theta] J)+M^{*} J=0
$$

Thus

$$
\begin{aligned}
\frac{\partial}{\partial \theta} \mathcal{H}( & A \sin \theta-B \cos \theta) \\
= & \frac{\partial}{\partial \theta}[(A \sin \theta-B \cos \theta) J] N J^{*}[A \sin \theta-B \cos \theta] \\
& +(A \sin \theta-B \cos \theta) J N \frac{\partial}{\partial \theta}\left(J^{*}[A \sin \theta-B \cos \theta]\right) \\
= & -M^{*} J N J^{*}[A \sin \theta-B \cos \theta]-[A \sin \theta-B \cos \theta] J N J^{*} M \\
= & -M^{*} \mathcal{H}^{*}-\mathcal{H} M=-2 \operatorname{Re} \mathcal{H} M .
\end{aligned}
$$

(3) At $\theta=\pi / 2$, for any function $\hat{v}$ obeying

$$
\hat{v}^{\mathrm{II}}(\lambda, \omega, s)-s \hat{v}^{\mathrm{II}}(\lambda, \omega, s)=e^{-\lambda} \hat{g}\left(e^{-\lambda}, \omega, s\right),
$$

there exists positive constants $\delta_{2}, K_{6}$, such that

$$
\begin{aligned}
\left(\hat{v}, \mathcal{H}\left(\pi^{\prime}, 2\right) \hat{v}\right) \geq & \delta_{2}\left(e^{\lambda / 2} \hat{v}, e^{\lambda / 2} \hat{\jmath}\right) \\
& -K_{6} \sum_{j=0}^{P_{2}}\left(e^{-\lambda / 2}(\partial / \partial \lambda)^{j} \hat{\hat{g}}, e^{-\lambda / 2}(\partial / \partial \lambda)^{j} \hat{g}\right)\left(1+|\omega|^{2}+|s|^{2}\right)^{Q_{1}} .
\end{aligned}
$$

At $\theta=0$, for any function $\hat{v}$ obeying

$$
(T \hat{v})^{\mathrm{III}}(\lambda, \omega, s)-R(T \hat{v})^{\mathrm{IV}}(\lambda, \omega, s)=e^{-\lambda} \hat{b}\left(e^{-\lambda}, \omega, s\right),
$$

there exist positive constants $\delta_{3}, K_{7}$, such that

$$
\begin{aligned}
(\hat{v}, \mathcal{H}(0) B \hat{v})> & \delta_{3}\left(e^{\lambda / 2} \hat{v}, e^{\lambda / 2} \hat{\imath}\right) \\
& -K_{7} \sum_{j=0}^{2}\left(e^{-\lambda / 2}(\partial / \partial \lambda)^{j} \hat{b}, e^{-\lambda / 2}(\partial / \partial \lambda)^{j} \hat{b}\right)\left(1+|\omega|^{2}+|s|^{2}\right)^{Q_{2}} .
\end{aligned}
$$

III. Proof of Main Theorem I using the symmetrizer. We begin by considering the auxiliary problem

$$
A \hat{v}_{x}+B \hat{v}_{y}+(C i \omega-s) \hat{v}=\hat{F}, \quad x, y \geq 0,
$$

with the boundary conditions

$$
\hat{v}^{\mathrm{I}}(0, y, \omega, s) \equiv 0, \quad(T \hat{v})^{\mathrm{III}}(y, 0, \omega, s)=0 .
$$


If we inner product the equation with $\hat{v}$, integrate over $x, y \geq 0$, and take the real part of both sides, we have

$$
(\eta-\epsilon)\|\hat{v}\|_{0,0}^{2}+K_{8}\left|\hat{v}_{B_{1}}\right|_{0,0}^{2}+K_{9}\left|\hat{v}_{B_{2}}\right|_{0,0}^{2} \leq K(\epsilon)\|F\|_{0,0}^{2} .
$$

Next, multiply (3.1) by $r(\partial / \partial r)$, and use the fact that

$$
r \frac{\partial}{\partial r} \frac{\partial}{\partial x}=\frac{\partial}{\partial x} r \frac{\partial}{\partial r}-\frac{\partial}{\partial x}, \quad r \frac{\partial}{\partial r} \frac{\partial}{\partial y}=\frac{\partial}{\partial y} r \frac{\partial}{\partial r}-\frac{\partial}{\partial y} .
$$

Let $(r(\partial / \partial r))^{j} \hat{v}=\hat{v}^{(j)}$, etc.

If $j=1$, we have

$$
A \hat{v}_{x}^{(1)}+B \hat{v}_{y}^{(1)}+(C i \omega-s) \hat{v}^{(1)}=\hat{F}^{(1)}+A \hat{v}_{x}+B \hat{v}_{y}=\hat{F}^{(1)}+\hat{F}-(C i \omega-s) \hat{v}
$$

$\hat{v}^{(1)}$ satisfies the same boundary condition as $\hat{v}$. Thus, we can obtain the a priori estimate

$$
\begin{aligned}
(\eta-\epsilon)\left\|\hat{\nu}^{(1)}\right\|_{0,0}^{2}+K_{8}\left|\hat{\nu}_{B_{1}}^{(1)}\right|_{0,0}^{2}+K_{9}\left|\hat{\nu}_{B_{2}}^{(1)}\right|_{0,0}^{2} \\
\quad \leq K(\epsilon)\left[\left\|\hat{F}^{(1)}\right\|_{0,0}^{2}+\|\hat{F}\|_{0,1}^{2}\right]+K_{1}(\epsilon)\|\hat{\nu}\|_{0,0}^{2} \\
\leq K(\epsilon)\left\|\hat{F}^{(1)}\right\|_{0,0}^{2}+K_{1}(\epsilon, \eta)\|\hat{F}\|_{0,1}^{2} .
\end{aligned}
$$

We may proceed inductively in order to obtain, for any $j$, the estimate

$$
(\eta-\epsilon)\left\|\hat{v}^{(j)}\right\|_{0,0}^{2}+K_{8}\left|\hat{v}_{B_{1}}^{(j)}\right|_{0,0}^{2}+K_{9}\left|\hat{v}_{B_{2}}^{(j)}\right|_{0,0}^{2} \leq K^{(j)}(\epsilon, \eta)\|\hat{F}\|_{j, j}^{2} .
$$

A similar technique works for the adjoint problem:

$$
A \hat{v}_{x}+B \hat{v}_{y}-[+C i \omega+\bar{s}] \hat{\imath}=\hat{F}
$$

with the boundary conditions

$$
\hat{v}^{\mathrm{II}}(0, y, \omega, s)=0, \quad(T \hat{v})^{\mathrm{IV}}(x, 0, \omega, s)=0 .
$$

The work of L. Sarason on weak = strong [13], these a priori inequalities, and Assumption 2.4 guarantee the existence of a strong solution, $\hat{v}$, to (3.1) for which these estimates are valid.

Thus, we consider the function

$$
\hat{w}=\hat{u}-\hat{v}
$$

$w$ satisfies

$$
\begin{aligned}
& \text { (a) } A \hat{w}_{x}+B \hat{w}_{y}+(C i \omega-s) \hat{w}=0 \\
& \text { (b) } \hat{w}_{B_{1}}^{\mathrm{I}}-S \hat{w}_{B_{1}}^{\mathrm{II}}=-\hat{v}_{B_{1}}^{\mathrm{I}}+S \hat{v}_{B_{1}}^{\mathrm{II}}+\hat{g}=\hat{g}_{1}, \\
& \text { (c) }\left(T \hat{w}_{B_{2}}\right)^{\mathrm{III}}-R\left(T \hat{w}_{B_{2}}\right)^{\mathrm{IV}}=-\left(T \hat{v}_{B_{2}}\right)^{\mathrm{III}}+R\left(T \hat{v}_{B_{2}}\right)^{\mathrm{IV}}+\hat{b}=\hat{b}_{1} .
\end{aligned}
$$

Again, we inner product equation (a) with $\hat{w}$, and integrate over $x, y \geq 0$. We thus obtain, taking real parts, 


$$
(\eta)\|\hat{w}\|_{0,0}^{2} \leq K_{10}\left|\hat{w}_{B_{1}}\right|_{0,0}^{2}+K_{11}\left|\hat{w}_{B_{2}}\right|_{0,0}^{2} .
$$

If we can obtain the estimate

$$
\left|\hat{w}_{B_{1}}\right|_{0,0}^{2}+\left|\hat{w}_{B_{2}}\right|_{0,0}^{2} \leq K_{12}\left(\left|\hat{v}_{B_{1}}\right| \widetilde{\widetilde{P}}_{1} \widetilde{Q}_{1}+|\hat{g}|_{\widetilde{P}_{1}, \widetilde{Q}_{1}}^{2}+\left|\hat{v}_{B_{2}}\right| \widetilde{\widetilde{P}}_{2}, \widetilde{Q}_{2}+|\hat{b}|_{\widetilde{P}_{2}, \widetilde{Q}_{2}}^{2}\right),
$$

then we can use the estimates in (3.7) to obtain an estimate for $\hat{w}$ of the type sought for $\hat{u}$. Finally, we add this to (3.3) to obtain the desired estimate for $\hat{u}$.

All we now need do is to obtain estimate (3.13) for $\hat{w}$. We know that $e^{-\lambda} \hat{w}\left(e^{-\lambda}, \theta, \omega, s\right)=\hat{p}(\lambda, \theta, \omega, s)$ satisfies

$$
\text { (a) } \begin{aligned}
(A \sin \theta- & B \cos \theta) \hat{p}_{\theta}+[A \cos \theta+B \sin \theta] \hat{p}_{\lambda} \\
+ & {\left[A \cos \theta+i 3 \sin \theta+\left[s-(i(1)] e^{-\lambda}\right] \hat{p}=0 .\right.}
\end{aligned}
$$

(b) $\hat{p}^{\mathrm{I}}(\lambda, \pi / 2, \omega, s) \ldots \mathrm{S} \hat{p}^{\mathrm{II}}(\lambda, \pi / 2,(1), s) \cdots c^{\lambda} \hat{g}_{1}\left(c^{-\lambda}, \omega, s\right)$,

(c) $(T \hat{p})^{\mathrm{III}}(\lambda, \cap,(1), s)-R(T \hat{p})^{\mathrm{IV}}(\lambda, 0, \omega, s)=e^{-\lambda} \hat{b}_{1}\left(e^{-\lambda}, \omega, s\right)$.

Multiply equation $(3.14)(a)$ by $\mathcal{H}$, then inner product with $\hat{p}$ and integrate $d \theta d \lambda, \theta$ from 0 to $\pi / 2, \lambda$ from $-\infty$ to $\infty$, and take the real part. Notice

$$
\begin{aligned}
((p, \mathcal{H}(\theta)(A \sin & \left.\left.\theta-B \cos \theta) \hat{p}_{\theta}\right)\right) \\
= & ((\hat{p},(\partial / \partial \theta)[(\mathcal{H}(\theta)(A \sin \theta-B \cos \theta)) \hat{p}])) \\
& -((\hat{p},[(\partial / \partial \theta)(\mathcal{H}(\theta)(A \sin \theta-B \cos \theta))] \hat{p})) \\
- & \left(\hat{p}_{B_{1}}, \mathcal{H}(\pi / 2) \Lambda \hat{p}_{B_{1}}\right)+\left(\hat{p}_{B_{2}}, \mathcal{H}(0) B \hat{p}_{B_{2}}\right) \\
& -\left(\left(\left(\hat{p}, \mathcal{H}(\theta)[\Lambda \sin O-B \cos \theta] \hat{p}_{\theta}\right)\right)\right. \\
& -((\hat{p},(\partial / \partial \theta)(\mathcal{H}(O)(\Lambda \sin O-B \cos O)) \hat{p})) .
\end{aligned}
$$

Thus

$$
\begin{aligned}
\operatorname{Re}((\hat{p}, \mathcal{H}(\theta) & \left.\left.(\Lambda \sin 0-B \cos \theta) \hat{p}_{\theta}\right)\right) \\
= & 1 / 2\left(\hat{p}_{B_{1}}, \mathcal{H}(\pi / 2) \Lambda \hat{p}_{B_{1}}\right)+1 / 2\left(\hat{p}_{B_{2}}, \mathcal{H}(0) B \hat{p}_{B_{2}}\right) \\
& -((\hat{p}, 1 / 2(\partial / \partial \theta)(\mathcal{H}(\Lambda \sin \theta-B \cos \theta)) \hat{p})) .
\end{aligned}
$$

Thus, if we add this to

$$
-\operatorname{Re}(\hat{p}, \mathcal{H} M \hat{p}),
$$

we get, using (2.34),

$$
\left(\hat{p}_{B_{1}}, \mathcal{H}\left((\pi / 2) \wedge \hat{p}_{B_{1}}\right)+\left(\hat{p}_{B_{2}}, \mathcal{H}(0) B \hat{p}_{B_{2}}\right)=0 .\right.
$$

We then use (2.38), (2.39) and merely replace $\lambda$ by $-\ln r$, with $\hat{p}(\lambda, \theta, \omega, s)=$ $e^{-\lambda} \hat{w}\left(e^{-\lambda}, \theta, \omega, s\right)$. The result is immediate. 
Thus we need only to construct the symmetrizer $\mathfrak{H}(\theta, \omega, s)$.

IV. Construction of the symmetrizer.

$$
M^{*}(\theta, \omega, s)=\left[(A \cos \theta+B \sin \theta)((\partial / \partial \lambda)-1)-(\bar{s}+C i \omega) e^{-\lambda}\right] .
$$

We are seeking an operator valued function of $\theta, \omega$ and $s$ which obeys (2.36)(b). Thus we let the operator equation in (b) operate on a function $\hat{v}(\lambda)$.

$$
J \hat{v}(\lambda)=\hat{g}(\lambda, \theta, \omega, s) .
$$

Then (2.36) (b) becomes

$$
\begin{aligned}
(A \cos \theta & +B \sin \theta) \hat{g}+(A \sin \theta-B \cos \theta) \partial \hat{g} / \partial \theta \\
& +(A \cos \theta+B \sin \theta) \partial \hat{g} / \partial \lambda-(A \cos \theta+B \sin \theta) g \\
& -(\bar{s}+C i \omega) e^{-\lambda} \hat{g}=0 .
\end{aligned}
$$

If we revert back to $(x, y)$ coordinates, we have $g$ satisfying

$$
A \hat{g}_{x}+B \hat{g}_{y}+[\bar{s}+C i \omega] \hat{g}=0 .
$$

We are now concerned with obtaining solutions to this homogeneous equation which are bounded in $x, y \geq 0$ uniformly in $\bar{s}$ and $\omega$. For this we need the following technical lemma.

(4.1) Lemma. If (2.1) is a strictly byperbolic system, then there exists a uniformly bounded matrix function $U\left(\omega_{2}, s, \omega\right)$ which is $C^{\infty}$ in its variables for $\eta \geq K>0$, all $\omega_{2}, \omega$, and with an inverse of the same type, such that $\exists$ a constant $\delta_{1}>0$ with

$$
U^{-1} A^{-1}\left(B i \omega_{2}+\bar{s}+C i \omega\right) U=\left(\begin{array}{cc}
L_{11} & L_{12} \\
0 & L_{22}
\end{array}\right),
$$

where $\operatorname{Re} L_{11} \geq \delta_{1} \eta$, Re $L_{22} \leq-\delta_{1} \eta$.

An analogous statement was formulated in Assumption 2.3 for $B^{-1}\left(A i \omega_{1}+\bar{s}+C i \omega\right)$ and is, of course, also valid.

Proof. Let $\zeta=\left(\omega_{2}, \xi, \omega\right)$. It is clear that for $|\zeta| \leq 1$ there exists a $C^{\infty}$ transformation $U$, such that

$$
U^{-1} A^{-1}\left(B i \omega_{2}+\bar{s}+C i \omega\right) U=\left(\begin{array}{cc}
L_{11} & 0 \\
0 & L_{22}
\end{array}\right)
$$

with the $L$ 's having the appropriate bounds. We shall consider $|\zeta| \geq 1 / 2$, then piece it together. Following Kreiss [6], we replace 


$$
\begin{aligned}
& M_{1}=A^{-1}\left(B i \omega_{2}+\bar{s}+C i \omega\right)=|\zeta| M_{1}\left(\zeta^{\prime}, \eta^{\prime}\right)=|\zeta| M_{1}^{\prime} \\
& \zeta^{\prime}=\zeta /|\zeta|, \quad \eta^{\prime}=\eta /|\zeta| .
\end{aligned}
$$

We can construct a $U$ for $\eta \geq \eta_{0}^{\prime}>0$, with $\eta_{0}^{\prime}$ any positive constant. Thus we need only consider $U$ in the neighborhood of every point $\zeta^{\prime}=\zeta_{0}^{\prime}, \eta^{\prime}=0$. Kreiss [6] and Ralst on [10] have shown that there exists a smooth $W$, with

$$
W^{-1} M_{1}^{\prime} W=\left(\begin{array}{cccc}
N_{11}^{\prime} & & & 0 \\
& N_{12}^{\prime} & & \\
& & M_{2}^{\prime} & \\
& & \ddots & \\
& & & M_{R}^{\prime}
\end{array}\right)
$$

where $\operatorname{Re} N_{12}^{\prime} \leq-2 \delta_{1} \eta, \operatorname{Re} N_{11}^{\prime}>2 \delta_{1} \eta ;$ for $\eta=0, \zeta^{\prime}=\zeta_{0}^{\prime}, j \geq 2$

$$
M_{j}\left(\zeta_{0}^{\prime}, 0\right)=\left(\begin{array}{ccc}
k_{j}^{\prime} & i & \\
& \ddots & \\
& \ddots & i \\
& & k_{j}^{\prime}
\end{array}\right) \text {, }
$$

$k_{j}^{\prime}$ is purely imaginary, $k_{j}^{\prime} \neq k_{l}^{\prime}$ if $j \neq l$,

$$
M_{j}\left(\zeta^{\prime}, \eta^{\prime}\right)=M_{j}\left(\zeta_{0}^{\prime}, 0\right)+\eta^{\prime} N_{j}\left(\zeta^{\prime}\right)+O\left(\left(\eta^{\prime}\right)^{2}\right)+E_{j}\left(\zeta^{\prime}\right),
$$

where $E_{j}\left(\zeta^{\prime}\right)$ has purely imaginary entries, $E_{j}\left(\zeta_{0}^{\prime}\right)=0$, and the element in the lower left corner of $N_{j}\left(\zeta^{\prime}\right)$, which we call $n_{s 1}$, has nonzero real part.

For each block $M_{j}^{\prime}$, consider the characteristic equation

$$
\begin{aligned}
\operatorname{Det}\left(M_{j}^{\prime}-k^{\prime}\right)= & \operatorname{Det}\left(M_{j}^{\prime}-k_{j}^{\prime}-\left(k^{\prime}-k_{j}^{\prime}\right)\right) \\
= & \left(k_{j}^{\prime}-k^{\prime}\right)^{s}+i^{s-1} \eta^{\prime} n_{s 1}+i^{s}\left(\zeta^{\prime}-\zeta_{0}^{\prime}\right) m_{s 1} \\
& +O\left(\left|\eta^{\prime}\right|^{2}+\left|\zeta^{\prime}-\zeta_{0}^{\prime}\right|^{2}\right),
\end{aligned}
$$

$\operatorname{Re} n_{s 1} \neq 0, \operatorname{Im} m_{s 1}=0$.

Thus the roots are

$$
k^{\prime}=k_{j}^{\prime}-\left[-i^{s-1} \eta^{\prime} n_{s 1}-i^{s}\left(\zeta^{\prime}-\zeta_{0}^{\prime}\right) m_{s 1}+O\left(\left|\eta^{\prime}\right|^{2}+\left|\zeta^{\prime}-\zeta_{0}^{\prime}\right|^{2}\right)\right]^{1 / s}
$$

This means that, if $\left|\zeta^{\prime}-\zeta_{0}^{\prime}\right| \leq k\left(\zeta_{0}^{\prime}\right)\left[\eta^{\prime}\right]^{1 / 2}$, the roots of $M_{j}$ split up with $1 / 2 s$, $1 / 2(s+1)$, or $1 / 2(s-1)$, having positive real part if $\eta^{\prime}>0$. The remainders then have negative real part.

Let

$$
\alpha=i\left[-i^{s-1} \eta^{\prime} n_{s 1}-i^{s}\left(\zeta^{\prime}-\zeta_{0}^{\prime}\right) m_{s 1}\right]^{1 / s} .
$$


Then the eigenvectors are

$$
\Phi_{j r}=\left(1, a, a^{2}, \cdots, a^{s-1}\right)^{T}+O\left(|\eta|^{2 / s}+\left|\zeta^{\prime}-\zeta_{0}^{\prime}\right|^{2 / s}\right)
$$

where for $r=1,2, \cdots, s$ different branches of a are taken.

We may use these eigenvectors to construct a bounded transformation $\widetilde{U}_{i}$ with a bounded inverse $\tilde{U}_{j}^{-1}$, both of which are smooth in the region $0 \leq \eta^{\prime} \leq k_{12}\left(\zeta_{0}^{\prime}\right)$, $\left|\zeta^{\prime}-\zeta_{0}^{\prime}\right| \leq k_{13}\left(\zeta_{0}^{\prime}\right)$, except for an algebraic branch point at $\zeta^{\prime}=\zeta_{0}^{\prime}, \eta^{\prime}=0$, such that

$$
\widetilde{U}_{j}^{-1} M_{j} \widetilde{U}_{j}=\left(\begin{array}{cc}
M_{11}^{(j)} & M_{12}^{(j)} \\
0 & M_{22}^{(j)}
\end{array}\right),
$$

$\operatorname{Re} M_{11}^{(j)} \geq 2 \delta_{1} \eta, \operatorname{Re} M_{22}^{(j)} \leq-2 \delta_{1} \eta$

We may do this for each block and for each $\zeta_{0}^{\prime}$. The result then follows by the compactness of the unit ball $\left|\zeta^{\prime}\right|=1$. Q.E.D.

We may now use this lemma in order to obtain the solutions to (4.4). Fourier transform (4.4) with respect to $y$, multiply by $A^{-1}$, then make the change of variables $\hat{g}=U \hat{b}$. Then (4.4) becomes

$$
\hat{b}_{x}+\left(\begin{array}{cc}
L_{11} & L_{12} \\
0 & L_{22}
\end{array}\right) \hat{b}=0,
$$

hence

$$
\hat{b}=\left[\begin{array}{c}
\exp \left(-L_{11} x\right) \hat{b}_{1} \\
0
\end{array}\right],
$$

where $\hat{b}_{1}$ is some $(m-l)$ vector valued function of $\left(\omega_{2}, s, \omega\right)$. We define

$$
\hat{g}_{1}=F_{\omega_{2}-y}^{-1} U\left[\begin{array}{c}
\exp \left(-L_{11} x\right) \hat{b}_{1} \\
0
\end{array}\right]
$$

as a function which obeys (4.4).

We may proceed in the same way and obtain

$$
\hat{g}_{2}=F_{\omega_{1}-x}^{-1} V\left[\begin{array}{c}
\exp \left(-K_{11} y\right) \hat{b}_{2} \\
0
\end{array}\right],
$$

where $\hat{b}_{2}$ is some $(m-p)$ vector valued function of $\left(\omega_{1}, s, \omega\right), \hat{g}_{2}$ is a vector valued function obeying (4.4). We now switch back to $\lambda, \theta$ coordinates and obtain

$$
\begin{aligned}
g_{1}= & \frac{1}{2 \pi} \int_{-\infty}^{\infty} \exp \left(i \omega_{2} e^{-\lambda} \sin \theta\right) d r_{2} \\
& \cdot l\left(r_{2}\right)\left[\begin{array}{c}
\exp \left(-L_{11}\left(\omega_{2}\right) e^{-\lambda} \cos \theta\right) \hat{b}_{1}\left(\omega_{2}, s, \omega\right) \\
0
\end{array}\right]
\end{aligned}
$$


and view this as an operator valued function of $\theta$ acting on functions of $\lambda$ in $C(-\infty, \infty) \cap L_{2}(-\infty, \infty)$.

For any such function $\hat{v}(\lambda)$, let

$$
\hat{b}_{1}\left(\omega_{2}, \omega, s\right)=\int_{-\infty}^{\infty} \hat{v}_{\mathrm{II}}(\lambda) \exp \left(-i \omega_{2} \lambda\right) d \lambda .
$$

We may define

$$
\widetilde{J_{1}}(\theta, \omega, s, \cdot) \hat{v}=\int_{-\infty}^{\infty} k_{1}(\theta, \omega, s, \lambda, r) \hat{v}(r) d r
$$

where

$$
\begin{aligned}
& k_{1}(\theta, \omega, s, \lambda, r) \\
& \quad=\frac{1}{2 \pi} \int_{-\infty}^{\infty} d \omega_{2} U\left(\omega_{2}\right)\left[\begin{array}{ll}
0 & \exp \left(-L_{11} e^{-\lambda} \cos \theta+i \omega_{2}\left(e^{-\lambda} \sin \theta\right)\right) \\
0 & 0
\end{array}\right] .
\end{aligned}
$$

Finally, we have $\tilde{J}_{1}^{*}$ which is an integral operator of the same type. Its kernel is

$$
k_{1}^{*}(\theta, \omega, s, \lambda, r)
$$

$$
=\frac{1}{2 \pi} \int_{-\infty}^{\infty} d \omega_{2}\left[\begin{array}{cc}
0 & 0 \\
\exp \left(-L_{11}^{*} e^{-r} \cos \theta\right) & 0
\end{array}\right] U^{*}\left(\omega_{2}\right) \exp \left(i \omega_{2}\left(\lambda-e^{-r} \sin \theta\right)\right) .
$$

Proceeding in the same inanner for $\tilde{J}_{2}$, we may then obtain $\tilde{J}_{2}^{*}(\theta)$ which is an integral operator of the same type, with

$$
\begin{aligned}
& k_{2}^{*}(\theta, \omega, s, \lambda, r) \\
& \quad=\frac{1}{2 \pi} \int_{-\infty}^{\infty} d \omega_{2}\left[\begin{array}{cc}
\exp \left(-K_{11}^{*} e^{-r} \sin \theta\right) & 0 \\
0 & 0
\end{array}\right] V^{*}\left(\omega_{2}\right) \exp \left(i \omega_{2}\left(\lambda-e^{-r} \cos \theta\right)\right) .
\end{aligned}
$$

We must consider now

$$
\tilde{J}^{*}=\tilde{J}_{1}^{*}+\tilde{J}_{2}^{*}
$$

In this construction for $\tilde{J}_{1}^{*}$ and $\tilde{J}_{2}^{*}$ we notice that we may premultiply by any operator on $L_{2}(-\infty, \infty)$ independent of $\theta$, then the result still satisfies (2.36) (a). At $\theta=\pi / 2$, we consider

$$
\begin{aligned}
& \tilde{J}_{1}^{*}(\pi / 2) A \hat{v}=F_{\omega_{2} \rightarrow \lambda}^{-1} \int_{-\infty}^{\infty}\left[\begin{array}{ll}
0 & 0 \\
I & 0
\end{array}\right] U^{*}\left(\omega_{2}\right) \exp \left(i \omega_{2}\left(\lambda-e^{-r}\right)\right) A \hat{v}(r) d r, \\
& \tilde{J}_{2}^{*}(\pi / 2) A \hat{v}=F_{\omega_{2} \rightarrow \lambda}^{-1} \int_{-\infty}^{\infty}\left[\begin{array}{cc}
\exp \left(-K_{11}^{*} e^{-r}\right) & 0 \\
0 & 0
\end{array}\right] V^{*}\left(\omega_{2}\right) A \hat{v}(r) d r .
\end{aligned}
$$

We make the change of variables $r=-\ln x_{1}$. We then have, letting

$$
\hat{p}\left(x_{1}\right)=\frac{\hat{v}\left(-\ln x_{1}\right)}{x_{1}}, \quad x_{1} \geq 0, \quad \hat{p}\left(x_{1}\right)=0, \quad x_{1}<0,
$$




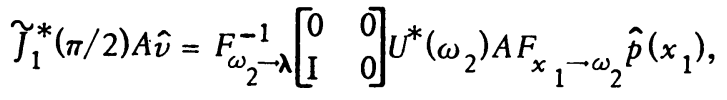

$$
\begin{aligned}
& \tilde{J}_{2}^{*}(\pi / 2) A \hat{v}=F_{\omega_{2} \rightarrow \lambda}^{-1} \int_{0}^{\infty}\left[\begin{array}{cc}
\exp \left(-K_{11}^{*}\left(\omega_{2}\right) x_{1}\right) d x_{1} & 0 \\
0 & 0
\end{array}\right] V^{*}\left(\omega_{2}\right) A \hat{p}\left(x_{1}\right) \text {. }
\end{aligned}
$$

At $\theta=0$, we have

$$
\begin{aligned}
& \tilde{J}_{1}^{*}(0) B \hat{v}=F_{\omega_{2} \rightarrow \lambda}^{-1} \int_{0}^{\infty}\left[\begin{array}{cc}
0 & 0 \\
\exp \left(-L_{11}^{*}\left(\omega_{2}\right) x_{1}\right) d x_{1} & 0
\end{array}\right] U^{*}\left(\omega_{2}\right) B \hat{p}\left(x_{1}\right), \\
& \tilde{J}_{2}^{*}(0) B \hat{v}=F_{\omega_{2} \rightarrow \lambda}^{-1}\left[\begin{array}{ll}
I & 0 \\
0 & 0
\end{array}\right] V^{*}\left(\omega_{2}\right) B F_{x_{1} \rightarrow \omega_{2}} \hat{p}\left(x_{1}\right) \text {. }
\end{aligned}
$$

In the expression (4.28) for $\tilde{J}_{1}^{*}(\pi / 2) A \hat{v}$, suppose

$$
p\left(x_{1}\right)=\left(\begin{array}{c}
S \Phi\left(x_{1}\right) \\
\Phi\left(x_{1}\right)
\end{array}\right) \text {. }
$$

Then

$$
F J_{1}^{*}(\pi / 2) A v=\left[\begin{array}{ll}
0 & 0 \\
I & 0
\end{array}\right] U^{*}\left(\omega_{2}\right) A\left[\begin{array}{l}
S \\
I
\end{array}\right] \Phi\left(\omega_{2}\right)=\left[\begin{array}{c}
0 \\
Q_{1}\left(\omega_{2}\right)
\end{array}\right] \Phi\left(\omega_{2}\right) .
$$

Kreiss' condition is exactly that the matrix $Q_{1}\left(\omega_{2}, \omega, s\right)$ is uniformly invertible for all $\omega_{2}, \omega, s, \operatorname{Re} s \geq 0$. Thus we let

$$
J_{1}^{*}(\theta)=P_{+} F^{-1}\left[\begin{array}{cc}
0 & 0 \\
0 & Q_{1}^{-1}\left(\omega_{2}, \omega, s\right)
\end{array}\right] F \widetilde{J}_{1}^{*}(\theta),
$$

where $P_{+}$is the orthogonal projection on $L_{2}(-\infty, \infty)$ defined by

$$
\begin{array}{ll}
P_{+} f\left(x_{1}\right)=f\left(x_{1}\right), & x_{1} \geq 0, \\
P_{+} f\left(x_{1}\right)=0, & x_{1}<0 .
\end{array}
$$

Next, in the expression (4.2) for $J_{2}^{*}(0) B \hat{v}$, suppose

$$
p\left(x_{1}\right)=T^{*}\left(\begin{array}{c}
R \phi\left(x_{1}\right) \\
\phi\left(x_{1}\right)
\end{array}\right) \text {. }
$$

We then have

$$
F \widetilde{J}_{2}^{*}(0) B u=\left[\begin{array}{ll}
I & 0 \\
0 & 0
\end{array}\right] V^{*}\left(\omega_{2}\right) B T^{*}\left(\begin{array}{c}
R \phi\left(x_{1}\right) \\
\phi\left(x_{1}\right)
\end{array}\right)=\left[\begin{array}{c}
Q_{2}\left(\omega_{2}\right) \\
0
\end{array}\right] \phi\left(x_{2}\right) .
$$

Again, Kreiss' condition is exactly that the matrix $Q_{2}\left(\omega_{2}, \omega, s\right)$ is uniformly invertible. Thus we let

$$
J_{2}^{*}(\theta)=P_{+} F^{-1}\left[\begin{array}{cc}
Q_{2}^{-1}\left(\omega_{2}, \omega, s\right) & 0 \\
0 & 0
\end{array}\right] F \hat{J}_{2}^{*}(\theta) .
$$


We now seek an operator $N$ such that if $p$ satisfies (4.30), then

$$
\left(J^{*}(\pi / 2) A v, N J^{*}(\pi / 2) A v\right) \geq \delta_{2}|p|_{0,0}^{2},
$$

and, if $p$ satisfies (4.34), then

$$
\left(J^{*}(0) B v, N J^{*}(0) B v\right) \leq-\delta_{2}|p|_{0,0}^{2} .
$$

We first discuss the operator $J_{2}^{*}(\pi / 2) A v$ for $p$ satisfying (4.30).

We solve the equation

$$
A u_{x}+B u_{y}+(C i \omega-s) u=A p(y) \delta(x-0),
$$

$$
y \geq 0, \quad(T u)^{\mathrm{III}}-R(T u)^{\mathrm{IV}}=0 \quad \text { at } y=0 .
$$

Fourier transform in $x$, multiply by $B^{-1}$

$$
\hat{u}_{y}+B^{-1}\left(A i \omega_{2}+C i \omega-s\right) \hat{u}=B^{-1} A p(y) .
$$

Then let $\hat{u}=B^{-1} V^{*-1} \tilde{u}$.

We have, using (2.25),

$$
\tilde{u}_{y}-\left(\begin{array}{cc}
K_{11}^{*} & 0 \\
K_{12}^{*} & K_{22}^{*}
\end{array}\right) \tilde{u}=V^{*} A p(y), \quad y \geq 0 .
$$

Thus

$$
\tilde{u}=\left(\begin{array}{c}
0 \\
\exp \left(K_{22}^{*} y\right) \tilde{u}_{\mathrm{III}}^{0}
\end{array}\right)+\left(\begin{array}{c}
-\int_{y}^{\infty} \exp \left(K_{11}^{*}(y-s)\right)\left[V^{*} A p(s)\right]_{\mathrm{IV}} d s \\
\int_{0}^{y} \exp \left(K_{22}^{*}(y-s)\right)\left[\left[V^{*} A p(s)\right]_{\mathrm{III}}+K_{12}^{*} \tilde{u}_{\mathrm{IV}}(s)\right] d s
\end{array}\right) .
$$

Kreiss' condition states that we can use the boundary conditions at $y=0$ to solve uniquely for $\tilde{u}_{\text {III }}^{0}$. Thus

$$
F \tilde{J}_{2}^{*}(\pi / 2) A \hat{v}=\left[\begin{array}{ll}
1 & 0 \\
0 & 0
\end{array}\right] V^{*} B U(0)
$$

But

$$
u(0)=T^{*}\left(\begin{array}{c}
R \psi \\
\psi
\end{array}\right)
$$

so finally, using (4.37),

$$
J_{2}^{*}(\pi / 2) A \hat{v}=\left(\begin{array}{c}
P_{+}(T u(x, 0, \omega, s))_{\mathrm{IV}} \\
0
\end{array}\right)=\left(\begin{array}{c}
T_{22} \Phi \\
0
\end{array}\right) .
$$

We may proceed in the analogous fashion to compute $J_{1}^{*}(0) B \hat{v}$ for $p\left(x_{1}\right)$ satisfying (4.35),

$$
J_{1}^{*}(0) B \hat{v}=\left(\begin{array}{c}
0 \\
P_{+} u(0, y, \omega, s)_{\mathrm{II}}
\end{array}\right)=\left(\begin{array}{c}
0 \\
T_{11} \psi
\end{array}\right) .
$$


Thus,

$$
T_{\omega, s}=T_{11} T_{22},
$$

where $T_{\omega, s}$ was defined in (2.17).

Let

$$
N=\left(\begin{array}{cc}
N_{11} & N_{12} \\
N_{12}^{*} & N_{22}
\end{array}\right) ; \quad N_{11}, N_{22} \text { are symmetric. }
$$

Equation (4.37) becomes

$$
T_{11}^{*} N_{11} T_{11}+2 \operatorname{Re} N_{12}^{*} T_{11}+N_{22} \geq \delta_{2}
$$

and (4.38) becomes

$$
-N_{11}-2 \operatorname{Re} N_{12} T_{22}-T_{22}^{*} N_{22} T_{22} \geq \delta_{2}
$$

Pre and post multiply (4.49) by $T_{22}^{*}$ and $T_{21}$ respectively and add to (4.50). We have

$$
T_{\omega, s}^{*} N_{11} T_{\omega, s}-N_{11}+2 \operatorname{Re} T_{22}^{*} N_{12}^{*}\left(T_{\omega, s}-1\right) \geq \delta_{2}\left(T_{22}^{*} T_{22}^{*}+1\right)
$$

Let

$$
N_{12}^{*}=\frac{\left(\delta_{2} T_{22}-T_{11}^{*} N_{11} T_{\omega, s}\right)}{2}\left(T_{\omega, s}-1\right)^{-1}
$$

and

$$
N_{11}=-\delta_{2}
$$

Thus

$$
\begin{aligned}
N_{21} & =\delta_{2}\left(I+T_{11}^{*} T_{11}\right)+\operatorname{Re} \delta_{2}\left(-T_{22}-T_{11}^{*} T_{\omega, s}\right)\left(T_{\omega, s}-1\right)^{-1} T_{11} \\
& =\delta_{2}\left[1-\operatorname{Re}\left(T_{22}+T_{11}^{*}\right)\left(T_{\omega, s}-1\right)^{-1} T_{11}\right],
\end{aligned}
$$

and

$$
\begin{aligned}
N_{12}^{*} & =\frac{\delta_{2}}{2}\left[T_{22}-T_{11}^{*} T_{\omega, s}\right]\left(T_{\omega, s}-1\right)^{-1} \\
& =\frac{\delta_{2}}{2}\left(\left[T_{22}-T_{11}^{*}\right]\left(T_{\omega, s}-1\right)^{-1}-T_{11}^{*}\right) .
\end{aligned}
$$

V. Verification of the estimates. We shall now verify conditions (1), (2) and (3) of Main Theorem II. We begin by noticing that the operator $T_{22}$ involves scalar operators of the form

$$
q\left(\omega_{2}, s, \omega\right) \int_{0}^{\infty} \exp \left(-\lambda\left(\omega_{2}, s, \omega\right) x\right) x^{j} f(x) d x,
$$


where $\lambda$ and $q$ are $C^{\infty}$ functions of all their variables if $s=\eta+i \xi, \eta \geq \eta_{0}>0$, except for certain algebraic branch points where multiple eigenvalues of

$$
B^{-1}\left(A i \omega_{2}-s+C i \omega\right)
$$

occur. However, because of (2.25), we have

$$
\left|x^{j} \exp \left(-\lambda\left(\omega_{2}, s, \omega\right) x\right) q\left(\omega_{2}, s, \omega\right)\right| \leq K_{1}^{1} \exp \left(-\delta_{1} \eta\right), \quad x \geq 0
$$

Let $x=1 / \operatorname{Re} \lambda\left(\omega_{2}, s, \omega\right)$; we then have

$$
\left|q\left(\omega_{2}, s, \omega\right)\right| \leq K_{2}^{l}\left[\operatorname{Re} \lambda\left(\omega_{2}, s, \omega\right)\right]^{j} .
$$

If we integrate (5.1) by parts $j$ times, we obtain

$$
\frac{q\left(\omega_{2}, s, \omega\right)}{\lambda^{j}\left(\omega_{2}, s, \omega\right)} \int_{0}^{\infty} \exp \left(-\lambda\left(\omega_{2}, s, \omega\right) x\right)\left(\frac{\partial}{\partial x}\right)^{j}\left(x^{j} f(x)\right) d x .
$$

We need now to estimate the operator

$$
\int_{0}^{\infty} \exp \left(-\lambda\left(\omega_{2}, s, \omega\right) x\right) f(x)=P_{\lambda} F f,
$$

i.e. we view this as an operator acting on the Fourier transform of $f$ :

$$
\int_{0}^{\infty} \exp \left(-i \omega_{2} x\right) f(x) d x=F f=\hat{f}\left(\omega_{2}\right)
$$

which takes $\omega_{2}$ into $-i \lambda\left(\omega_{2}, s, \omega\right)$. In order to estimate this, we shall transform the lower half plane into the unit circle and then use Theorem 1 of Carleson [1]. Notice

$$
U f=\hat{f}\left(i \frac{(z+1)}{(z-1)}\right) \frac{2^{1 / 2}}{(1-z)}
$$

is a unitary map of $L_{2}[0, \infty]$ one-to-one onto $H^{2}$ of the circle. We seek the transformation which takes $\hat{f}(i(z+1) /(z-1))(1 /(1-z))$ into $\hat{f}(-i \lambda(i(z+1) /(z-1)))(1 /(1-z))$. Let

$$
i(z)=\frac{i(z+1)}{(z-1)}, \quad p^{-1}(z)=\frac{z+i}{z-i} .
$$

Then the transformation in question is

$$
T g=g\left(p^{-1}(-i \lambda(p(z)))\right)\left(\left|\frac{p^{\prime}(z)}{p^{\prime}\left(p^{-1}(-i \lambda(p(z)))\right)}\right|\right)^{1 / 2} .
$$

The function $\lambda$ is an eigenvalue of $B^{-1}\left(A i \omega_{2}+C i \omega-s\right)$ with positive real part. We can show therefore, as $\omega_{2} \downarrow 0$ that the function $\lambda\left(1 / \omega_{2}\right)$ has a Puiseux expansion for some positive integer $p$. 


$$
\lambda\left(1 / \omega_{2}\right)=\frac{\lambda_{-1}}{\omega_{2}}+\sum_{j=1}^{\infty} \lambda_{j} \omega_{2}^{j / p-1}
$$

with $\lambda_{-1} \neq 0$.

We can differentiate this and obta in

$$
\lambda^{\prime}\left(\omega_{2}\right)=\lambda_{-1}+\sum_{j=1}^{\infty} \lambda_{j}(1-j / p)\left(\omega_{2}\right)^{-j / p} .
$$

Thus, the function $-i \lambda\left(\omega_{2}\right)$ is one-to-one if $\omega_{2} \geq M(s, \omega)$, also if $\omega_{2} \leq-M(s, \omega)$, where

$$
M(s, \omega) \leq M_{0}\left(1+|s|^{2}+|\omega|^{2}\right)^{Q_{1} / 2}
$$

for some $Q_{1} \geq 0$.

We shall only estimate the operator in (5.5) for $\omega_{2} \geq M$. This corresponds to bounding

$$
\int_{0}^{\theta_{1}}\left|f\left(p^{-1}\left(-i \lambda\left(p\left(e^{i \theta}\right)\right)\right)\right)\right|^{2} \frac{\left|p^{\prime}\left(e^{2 \theta}\right)\right|}{\left|p^{\prime}\left(p^{-1}\left(-i \lambda\left(p\left(e^{i \theta}\right)\right)\right)\right)\right|} d \theta
$$

for $\epsilon \geq \theta_{1}>0$. By the one-to-one property of $\lambda$, we may make the change of variables

$$
z=p^{-1}\left(-(i \lambda)^{-1}(p(\eta))\right)=e^{i \theta}, \quad 0 \leq 0 \leq \theta_{1},
$$

$$
|p(z) d z|=\frac{\left|p^{\prime}(\eta) a^{\prime} \eta\right|}{\left|\lambda^{\prime}\left((-i \lambda)^{-1}\left(p^{\prime}(\eta)\right)\right)\right|},
$$

so we must bound

$$
\int_{\Gamma}|f(\eta)|^{2} \frac{d \eta}{\left|\lambda^{\prime}\left((-i \lambda)^{-1}(p(\eta))\right)\right|},
$$

where $\Gamma$ is the curve defined by $(5.14)$

$$
\Gamma(\theta)=R(\theta) e^{i \phi(\theta)} .
$$

According to Carleson's result [1], we need only estimate

$$
\frac{1}{l} \int_{0}^{\min \left(R^{-1}(1-l), \phi^{-1}(l)\right)} \frac{\left(\left|R^{\prime}(\theta)\right|^{2}+|R(\theta)|^{2}\left(\phi^{\prime}(\theta)\right)^{2}\right)^{1 / 2}}{\mid \lambda^{\prime}\left((-i \lambda)^{-1}\left(p\left(R(\theta) e^{i \phi(\theta)}\right)\right) \mid\right.} d \theta .
$$

Thus, we shall have as a bound

$$
\sup \frac{1}{\left|\lambda^{\prime}\left(\omega_{2}, s, \omega\right)\right|} \text { for } \omega_{2} \geq M(\omega, s) .
$$

Similar reasoning works for $\omega_{2} \leq-M(\omega, s)$. This quantity can also be bounded by a constant multiple of $\left(1+|\omega|^{2}+|s|^{2}\right)^{Q_{2}}$. Thus we have shown 


$$
\left|T_{22} f\right|_{0,0}^{2} \leq K_{3}^{1}|f|_{22}^{2}, Q_{22}
$$

A similar argument works for $T_{11}$. We next consider $T_{22}^{*}$ which involves sums of scalar operators of the form

$$
x^{j} \int_{-\infty}^{\infty}-\exp \left(-\lambda\left(\omega_{2}, s, \omega\right) x\right) q\left(\omega_{2}, s, \omega\right) \hat{f}\left(\omega_{2}\right) d \omega_{2}, \quad x \geq 0,
$$

where $q$ obeys (5.3). We may replace

$$
\hat{f}\left(\omega_{2}\right)=\phi \hat{f}+(1-\phi) \hat{f}
$$

where $\phi\left(\omega_{2}: \omega, s\right)$ is in $C^{\infty}$,

$$
\begin{aligned}
& \phi \equiv 1 \quad \text { if }\left|\omega_{1}\right| \geq M(s, \omega)+1 \\
& \phi \equiv 0 \text { if }\left|\omega_{1}\right| \leq M(s, \omega), \quad 0 \leq \phi \leq 1 .
\end{aligned}
$$

The quantity involving $(1-\phi) \hat{f}$ can be easily estimated appropriately. We thus wish to estimate

$$
x^{j} \int_{M}^{\infty} \exp \left(-\lambda\left(\omega_{2}, s, \omega\right) x\right) q \hat{f}\left(\omega_{2}\right) d \omega_{2} .
$$

We may integrate by parts $j$ times, and obtain

$$
\int_{M}^{\infty} \exp \left(-\lambda\left(\omega_{2}, s, \omega\right) x\right)\left(\frac{\partial}{\partial \omega_{2}} \frac{1}{\lambda^{\prime}\left(\omega_{2}, s, \omega\right)}\right)^{j} q\left(\omega_{2}, s, \omega\right) \hat{f}\left(\omega_{2}\right) d \omega_{2} .
$$

We can use (5.3), (5.10) and (5.11) to obtain the same estimate as for $T_{22}$, modulo different $K_{3}^{1}$ and $Q_{22}$. Of course, a similar argument is valid for $T_{11}^{*}$.

In order to estimate products of these operators we must consider the effect of $(x(\partial / \partial x))^{r}$ on them. Because of Assumption (2.3) or Lemma (4.1), we may differentiate (5.1) with respect to $\omega_{2}$, and the effect will be only an increase in bound of the form $K_{3}\left(1+|\omega|^{2}+|s|^{2}\right)^{Q_{3 / 2}}$. Thus we are considering expressions of the form

(5.24) $\int_{0}^{\infty} d x \int_{-\infty}^{\infty} d \omega_{2} \exp \left(i \omega_{2} y-\lambda\left(\omega_{2}, s, \omega_{1}\right) x\right)\left(\omega_{2} y\right)^{j} q\left(\omega_{2}, s, \omega\right)\left(\frac{\partial^{r}}{\partial x}\left(x^{r} f(x)\right)\right) d x$, $y \geq 0$ and $q$ is uniformly bounded. Integrate by parts $j$ times with respect to $\omega_{2}$, we have (modulo constants)

$$
\int_{0}^{\infty} d x \int_{-\infty}^{\infty} \exp \left(i \omega_{2} y\right)\left(\frac{\partial}{\partial \omega_{2}}\right)^{j}\left[\omega_{2}^{j} \exp \left(-\lambda\left(\omega_{2}, s, \omega\right) x\right) q\left(\omega_{2}, s, \omega\right)\right]\left(\frac{\partial}{\partial x}\right)^{r}\left(x^{r} f(x)\right) .
$$

This becomes a sum of terms of the form

$$
\int_{0}^{\infty} d x \int_{-\infty}^{\infty} \exp \left(i \omega_{2} y-\lambda\left(\omega_{2}, s, \omega\right) x\right)\left[\omega_{2}^{p} x^{p} q_{p}\left(\omega_{2}, s, \omega\right)\right]\left(\frac{\partial}{\partial x}\right)^{r}\left(x^{r} f(x)\right) d x
$$


$p=0,1, \cdots, j$, where $q_{p}$ is bounded by $\left(1+|\omega|^{2}+|s|^{2}\right)^{Q_{0}^{(p)}} K_{0}^{(p)}$. Next integrate by parts $p$ times in $x$, recall that $\left(1+|\omega|^{2}+|s|^{2}\right)^{Q_{1}^{(p)}}>\omega_{2} / \lambda\left(\omega_{2}, s, \omega\right)$. We then have terms of the form

$$
\int_{0}^{\infty} \int_{-\infty}^{\infty} \exp \left(i \omega_{2} y-\lambda\left(\omega_{2}, s, \omega\right)\right) \widetilde{q}_{p}\left(\omega_{1}, s, \omega\right)\left(\frac{\partial}{\partial x}\right)^{(r+p)} x^{(r+p)} f(x) d x .
$$

We thus have shown

$$
\left|T_{22} f\right|_{P, Q}^{2} \leq K_{3}^{1}(P, Q)|f|_{P^{\prime}}^{2}(P, Q), Q^{\prime}(Q, P) \cdot
$$

Next, we apply $(x(\partial / \partial x))^{r}$ to $(5.20)$, obtaining terms of the form

$$
x^{r+j-p} \int_{-\infty}^{\infty} \exp \left(-\lambda\left(\omega_{2}, s, \omega\right) x\right) \lambda^{(r-p)} q\left(\omega_{2}, s, \omega\right) \hat{f}\left(\omega_{2}\right) d \omega_{2},
$$

then integrate by parts $r+j-p$ times in $\omega_{2}$. An estimate of the type (5.28) for $T_{22}^{*} f$ then follows. Similar arguments hold for $T_{11} f$ and $T_{11}^{*} f$.

We must also consider the $\theta$ dependent operators in order to verify condition (1) of Main Theorem II. This just becomes a matter of showing that terms of the form

$$
\left(\frac{\partial}{\partial \theta}\right)^{K} q\left(\omega_{2}, s, \omega\right) \int_{0}^{\infty} \exp \left(-\lambda\left(\omega_{2}, s, \omega\right) x \cos \theta+i \omega_{2} x \sin \theta\right)(\cos \theta)^{j} x^{j} f(x) d x
$$

exist as maps of $C^{\infty}(0, \infty) \cap L_{2}(0, \infty)$ into $L_{2}(-\infty, \infty)$.

If $\lambda\left(\omega_{2}\right) / i \omega_{2}$ does not approach an eigenvalue of $B^{-1} A$ which is positive and equals tangent $\theta$, then our previous integration by parts technique works.

This is indeed the case because of our "modified ellipticity" Assumption (2.4). This is the only place we need it in this work.

Condition (2) of Main Theorem II is valid because of our construction of the symmetrizer.

Condition (3) is valid if the boundary conditions are homogeneous. We must merely examine the effect of the inhomogeneities in the boundary terms. Thus we are considering

$$
(\hat{v}, H(\pi / 2) A \hat{v})
$$

for

$$
\begin{aligned}
& \hat{v}=\left(\begin{array}{c}
e^{-\lambda} \hat{g}\left(e^{-\lambda}\right) \\
0
\end{array}\right)+\left(\begin{array}{c}
S e^{-\lambda} p\left(e^{-\lambda}\right) \\
e^{-\lambda} p\left(e^{-\lambda}\right)
\end{array}\right), \\
& \hat{v}=\hat{v}_{g}+\hat{v}_{B} .
\end{aligned}
$$

Thus we have

$$
\left(\hat{v}_{B}, H(\pi / 2) A \hat{v}_{B}\right)+2 \operatorname{Re}\left(\hat{v}_{B}, H(\pi / 2) A \hat{v}_{g}\right)+\left(\hat{v}_{g}, H(\pi / 2) A \hat{v}_{g}\right) .
$$


We may now estimate the last two terms with the help of Schwarz' inequality, the estimates involving $T_{11}$ and $T_{22}$ and their adjoints and Assumption (2.5). The result follows in the same way at $\theta=0$.

VI. Comments. It is clear that we may estimate all norms of the solution $\|\hat{u}\|_{P, Q}\left|\hat{u}_{B}\right|_{P^{\prime}, Q^{\prime}}$ merely by multiplying (2.6) by $r(\partial / \partial r)$ and proceeding inductively, following the procedure used in the beginning of $\$ 3$. Moreover, we can then use the polar coordinate form of the equation to estimate the $\theta$ derivatives in terms of $L_{2}$ norms of $(r(\partial / \partial r))^{p}\left(1+|\omega|^{2}+|s|^{2}\right)^{Q_{r} P^{\prime}}$ times a lower $\theta$ derivative of $\hat{F}$ and $P^{\prime}, Q^{\prime}$ norms of positive powers of $r$ times $\hat{f}$ and $\hat{g}$.

The scalar wave equation

$$
\begin{aligned}
& u_{x x}+u_{y y}-u_{t t}=F, \quad x, y, t \geq 0, \\
& u(x, y, 0)=0, \\
& u_{t}(x, y, 0)=0, \\
& u_{x}-4 / 3 u_{y}=g(y), \quad x=0, \\
& u_{x}-4 / 3 u_{y}=b(x), \quad y=0,
\end{aligned}
$$

will admit no reasonable est imate, since

$$
e^{q(-4 x-3 y+5 t)}=u_{q}(x, y, t)
$$

will solve the equation with initial data approaching 0 as $q \rightarrow \infty$, while the solutions blow up for any positive $t$ in an exponential fashion as $q \rightarrow \infty$.

However, the reduced wave equation

$$
u_{x x}+u_{y y}-s^{2} u=F
$$

with the same boundary condition, and $s$ fixed, will, by the work of Kondrat'ev, admit certain reasonable a priori estimates modulo a finite number of eigenfunctions of the homogeneous equation. The difference, of course, is due to the fact that these estimates depend on $s$ and trouble develops as the imaginary part of $s$ becomes unbounded.

\section{BIBLIOGRAPHY}

1. L. Carleson, Interpolations by bounded analytic functions and the corona problem, Ann. of Math. (2) 76 (1962), 547-559. MR 25 \#5 186.

2. R. G. Douglas and R. Howe, On the $C^{*}$-algebra of Toeplitz operators on the quarter plane, Trans. Amer. Math. Soc. 158 (1971), 203-217.

3. R. Hersh, Mixed problems in several variables, J. Math. Mech. 12, (1963), 317334. MR $26 \# 5304$.

4. V. A. Kondrat'ev, Boundary-valuc problems for elliptic equations in conical regions, Dokl. Akad. Nauk SSSR 153 (1963), 17-29 = Soviet Math. Dokl. 4 (1963), 1600 1602. MR $28 \# 1383$. 
5. L. Kraus and L. Levine, Diffraction by an elliptic cone, Comm. Pure Appl. Math. 14 (1961), 49-68. MR 22 \#10554.

6. H.-O. Kreiss, Initial boundary valued problems for hyperbolic systems, Comm. Pure Appl. Math. 23 (1970), 277-298.

7. I. A. K. Kupka and S. J. Osher, On the wave equation in a multi-dimension corner, Comm. Pure Appl. Math. 24 (1971), 381-393.

8. Ja. B. Lopatinskii, On a method of reducing boundary problems for a system of differential equations of elliptic type to regular equations, Ukrain. Mat. Ž. 5 (1953), 123151. MR 17, 494.

9. A. S. Peters, Water waves over sloping beaches and the solution of a mixed boundary value problem for $\Delta^{2} \phi-K^{2} \phi-0$ in a sector, Comm. Pure Appl. Math. 5 (1952), 87-108. MR 13, 789.

10. J. Ralston, Note on a paper of Kreiss, Comm. Pure Appl. Math. 24 (1971).

11. J. Rauch, $\mathfrak{L}_{2}$ is a continuable initial condition for Kreiss' mixed problems, Comm. Pure Appl. Math. 25 (1972), 265-285.

12. R. Sakomoto, Mixed problems for hyperbolic equations. I, II, J. Math. Kyoto Univ. 10 (1970), 349-373, 403-417.

13. L. Sarason, On weak and strong solutions of boundary value problems, Comm. Pure Appl. Math. 15 (1962), 237-288. MR 27 \#66.

DEPARTMENT OF MATHEMATICS, STATE UNIVERSITY OF NEW YORK AT STONY BROOK, STONY BROOK, NEW YORK 11790 